\title{
Most cited 100 articles from Turkey on abdominal wall hernias: a bibliometric study
}

\author{
Hakan Kulaçoğlu' (D), Haydar Celasin²(D) \\ ${ }^{1}$ Department of Surgery, Ankara Hernia Center, Ankara, Turkey \\ ${ }^{2}$ Department of Surgery, Lokman Hekim Akay Hospital, Ankara, Turkey
}

\begin{abstract}
Objective: The aim of the present study was to search the most-cited articles from Turkey on abdominal wall hernias and analyze their characteristics with several parameters.

Material and Methods: In March 2019, a search was conducted through all databases in the Web of Science (WoS) to determine the most-cited articles on abdominal wall hernias. Each article was evaluated in regard to host journal, year of publication, the complete list of authors, the type of article, main subject of the study, institution of the study group. Citation counts in Google Scholar (GSch) were also obtained.

Results: Mean number of citations of the top 100 articles in herniology was 30.50. Articles were published in 38 journals; Hernia is the leading host. No correlation was observed between the journal impact factors and the number of the citations. Two thirds of the articles were clinical studies. Article types had no significant effect on the citation counts. Inguinal hernia was the most frequent topic by taking place in 58 papers. Articles related to incisional hernias had a higher mean number of citations in comparison with other topics. Ankara University School of Medicine had most cited articles, the highest number of total citations, and the highest citation per articles. Ankara Numune Training and Research Hospital and Istanbul University School of Medicine had the highest number of the articles in the list.
\end{abstract}

Conclusion: Citation counts of hernia related articles from Turkey are relatively low. Hernia is the leading journal for Turkish studies. Inguinal hernia is the most frequent topic whereas papers about incisional hernias receive more citations than others.

Keywords: Hernia, abdominal wall, bibliometric, citation

Cite this article as: Kulaçoğlu $\mathrm{H}$, Celasin H. Most cited 100 articles from Turkey on abdominal wall hernias: a bibliometric study. Turk J Surg 2020; 36 (2): 180-191

Corresponding Author

Hakan Kulaçoğlu

E-mail: hakankulacoglu@hotmail.com

Received: 23.05.2019

Accepted: 13.08 .2019

Available Online Date: 08.06 .2020

(C) Copyright 2020 by Turkish Surgical Society Available online at www.turkjsurg.com

DOI: $10.5578 /$ turkjsurg.4536

\section{INTRODUCTION}

The number of publications from Turkey displays an obvious growth as biomedical publishing advances globally (1). However, recently Onat has performed a citation analysis and stated that Turkey's contribution to the medicine by scientific articles is not enough compared with the potential of the country (2). Citation (a reference to subsequent studies) in another paper is one of the criteria to value an article. Citation analysis of the publications in a specific subject is performed by bibliometric methodology. The earliest example was published in the Journal of the American Medical Association as one of the most-cited articles in the same journal in 1985 (3). In 2002, a study on 100 citation classics in general surgery journals was published (4). Mayir et al. have searched the most cited articles from Turkey in the general surgery field and revealed that hydatid disease, pilonidal sinus, breast diseases, and inguinal hernia were the most frequent subjects (5). Scientific papers on abdominal wall hernias also show a steady rise worldwide (6). Hernia repairs, especially those for inguinal hernias, can be performed in every institution, and the surgeons find opportunity to prepare scientific papers more easily in comparison with major operations in surgical practice.

The present study was done with the purpose of listing the most-cited articles from Turkey in the field of abdominal wall hernia and analyzing their characteristics with several parameters. 


\section{MATERIAL and METHODS}

\section{Database Search}

In March 2019, a search was conducted through all databases in the Web of Science (WoS) to determine the most-cited articles on abdominal wall hernias from Turkey. The keywords for the topic line of the search were "inguinal hernia," "ventral hernia," "incisional hernia," "umbilical hernia," "paraumbilical hernia," and "femoral hernia." Additionally, combinations of "hernia and emergency," "hernia and mesh" were added. The keywords were searched in the titles, abstracts, and keywords given.

\section{Data Recording}

The study adhered to the Helsinki Declaration developed by the World Medical Association for medical research involving human material and data. After the top-cited 100 articles were determined and ranked by the number of citations, all full texts were reached. Each article was evaluated in regard to journal name, main field of the journal (surgery, general medicine, others), year of publication, the complete list of authors, number of authors, the type of article (clinical study; review/systematic review/meta-analysis/literature search; case report/case series; laboratory study/animal experiment/cadaver dissection), main subject of the study (inguinal, incisional, umbilical, femoral, etc; emergency; mesh and other materials - if the study focused on the characteristics or properties of prosthetic materials, or if several meshes were compared in repairing certain hernia types), institution of the study group. Citation counts in Google Scholar (GSch) were also obtained. The data were recorded in Office Excel 2016 (Microsoft, Redmond, WA).

\section{Statistical Analysis}

Data were exported to SPSS v.21 (IBM, Chicago, IL) for statistical analysis. A one-way ANOVA test was used to determine the differences between mean values. A correlation coefficient ( $r$ ) was calculated to determine whether recorded parameters correlated with the citation counts of the listed articles. A p value less than 0.05 was accepted as significant.

\section{RESULTS}

The top 100 list of hernia related papers from Turkey is given in Table 1. The total number of citations of the top 100 articles in herniology was 3.050 in WoS (range: 12-145), and 5.672 in GSch (range: 16-272). Mean number of citations was 30.50 in WoS, and 56.72 in GSch.

The publication year was evaluated in 3 consecutive decades (Table 2). No article published before 1995 or earlier took place in the list. The most productive decade was 2000-2009, with 72 papers. The year with the highest number of articles in the list was 2006 (14 papers). The most recent paper in the list was published in 2014. No effect of the publication decade was observed on citation counts (Table 3 ).
One hundred top cited articles were published in 38 different journals: 80 papers in surgical journals and 20 papers in others (anesthesia, biomaterials, etc.). Hernia was the most frequent host journal with 23 articles (Table 4). Articles published in surgical journals had more citations than the ones in the journals from other disciplines (WoS: 32.85 vs. 21.10; $p=0.030$, and GSch: 60.70 vs. $40.80 ; p=0.57)$. No correlation was observed between the journal impact factors and the number of the citations. The number of the authors differed 1 to 11 with a mean number of 5.66; only 3 papers were written by one single author, and 2 by two authors. There was no correlation between the number of the authors and the citation counts. Interestingly, four papers had no authors from general surgery at all; two by anesthesiologists, one by radiologists, and another one by neurologists and urologist.

Two thirds of the articles were clinical studies. The types of the articles had no significant effect on citation counts (Table 5). Amongst the clinical studies, retrospective series had more citation counts than prospective studies (WoS: 44.38 vs. 27.76; $p=$ 0.007, and GSch: 85.43 vs. 51.47; $p=0.011$ ).

Inguinal hernia was the most frequent topic by taking place in 58 papers, incisional hernia was the second with 16 studies (Table 6). Twenty-one articles studied mesh materials. Six studies which investigated hernia repairs in emergency conditions had the highest mean citation number (WoS: 95.50; GSch: 177.50). Articles related to incisional hernias had a higher mean number of citations in comparison to ones about inguinal hernias (WoS: 38.73 vs. $28.12 ; p=0.080$ ).

The top 100 articles originated from 43 institutions. There were 29 university hospitals, 10 teaching hospitals other than medical schools, 3 private hospitals and 1 rural public hospital. From another view, there were 39 publications from Ankara, 22 from Istanbul, 11 from Izmir and 28 from other cities. Ankara University School of Medicine had the most-cited article, the highest number of total citations, and the highest citation counts per articles. Ankara Numune Training and Research Hospital and Istanbul University School of Medicine had the highest number of the articles in the list (Table 7). On the other hand, no differences in citation counts were determined in comparison of university hospitals and other training hospitals.

\section{DISCUSSION}

Bibliometric analyses for citation counts in medical publishing have revealed that the most-cited articles were published between 1990 and 2010 (7-12). The most productive decade in the present study was 2000-2009. Even the most scientific articles need time to get citations by subsequent publications. Citation count lists are dynamic, and rankings may change by time, and we can see a rapid rise in the citation counts of papers produced in the last decade. Similarly, some articles that are not yet in the 


\begin{tabular}{|c|c|c|c|c|c|c|c|c|c|c|c|c|c|c|c|c|c|}
\hline 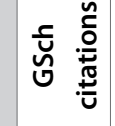 & $\stackrel{\mathbb{N}}{\nwarrow}$ & $\stackrel{\tilde{N}}{\stackrel{\sim}{n}}$ & $\stackrel{J}{ \pm}$ & $\stackrel{0}{\circ}$ & $\stackrel{\widetilde{\Omega}}{\sim}$ & $\cong$ & $\cong$ & $\stackrel{\text { t }}{\circ}$ & $\underline{6}$ & g & $\infty$ & $\stackrel{\odot}{ }$ & $\stackrel{\infty}{\curvearrowright}$ & $\begin{array}{l}\infty \\
\infty\end{array}$ & ৪ & $\bar{\lambda}$ & $\stackrel{m}{\curvearrowright}$ \\
\hline 号 & $\stackrel{\text { qu }}{\square}$ & $\stackrel{d}{\stackrel{0}{0}}$ & $\bar{\sigma}$ & $\infty$ & $\infty$ & $\hat{\sigma}$ & 6 & 告 & t & $\widetilde{\sigma}$ & $\stackrel{\infty}{\leftrightarrow}$ & in & $\stackrel{n}{n}$ & 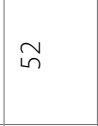 & in & \& & fo \\
\hline 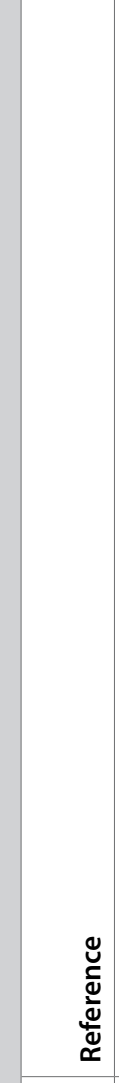 & 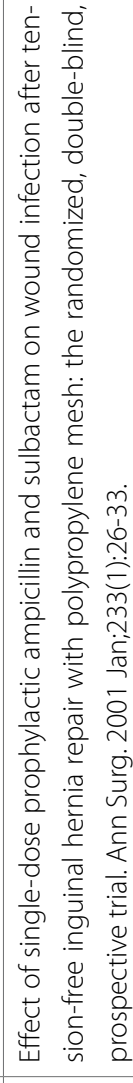 & 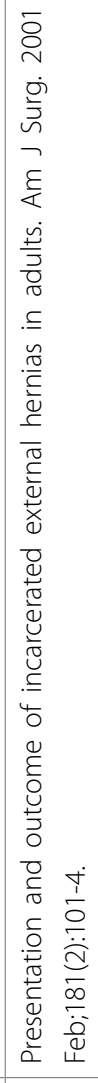 & 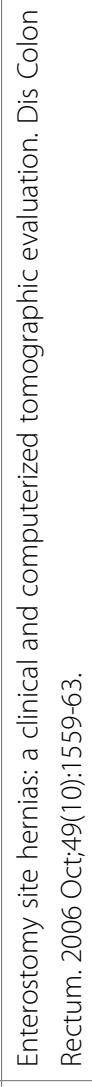 & 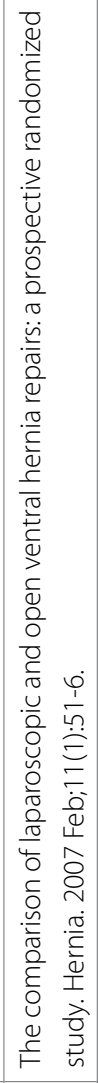 & 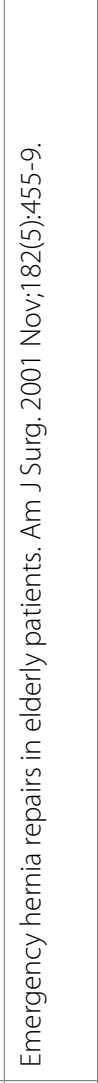 & 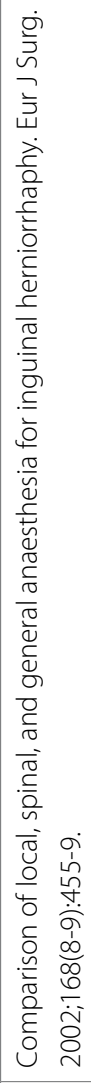 & 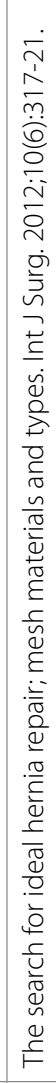 & 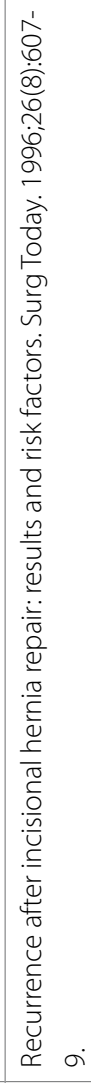 & 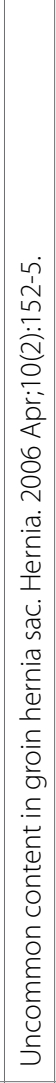 & 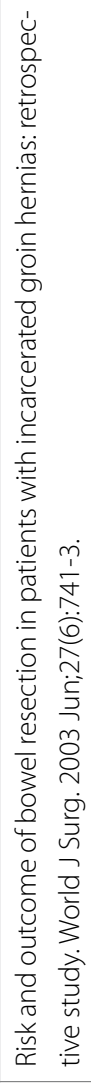 & 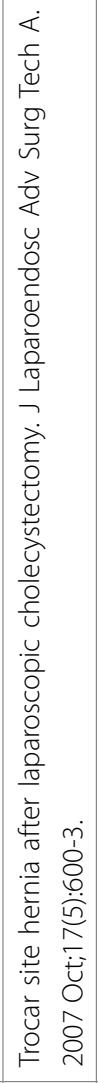 & 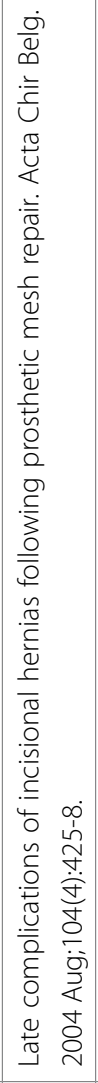 & 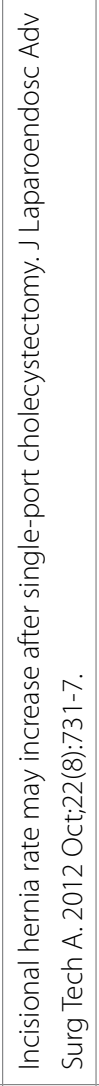 & 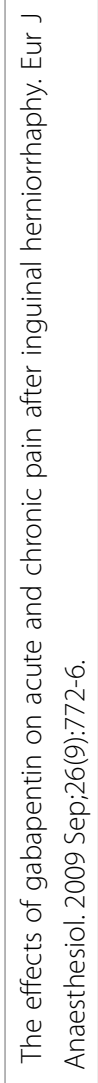 & 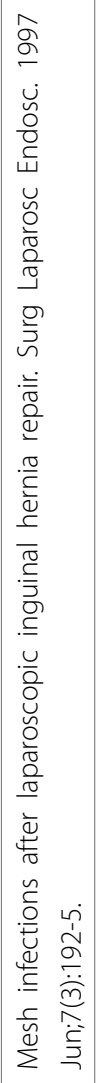 & 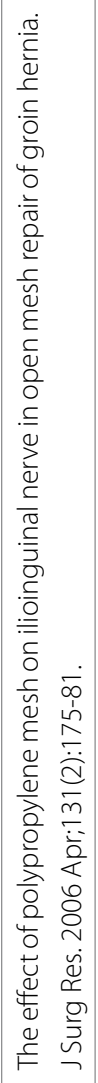 & 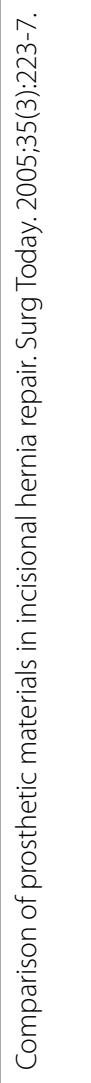 \\
\hline 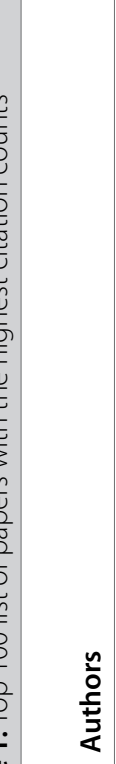 & 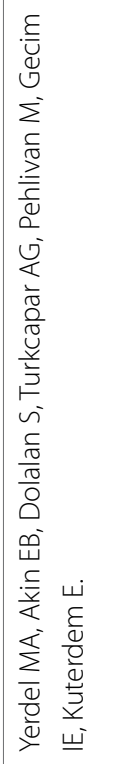 & 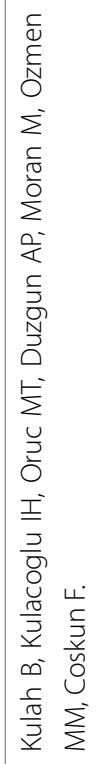 & 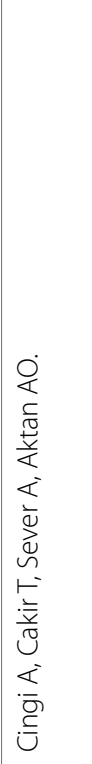 & 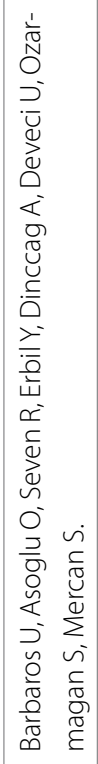 & 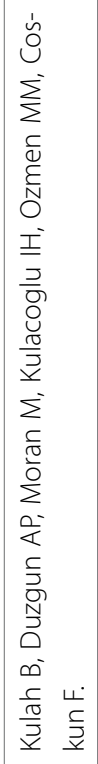 & 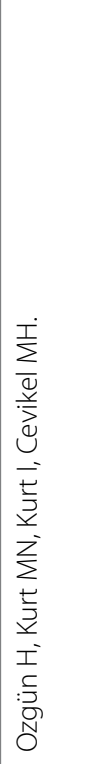 & 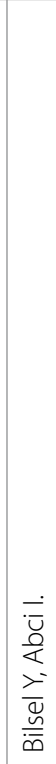 & 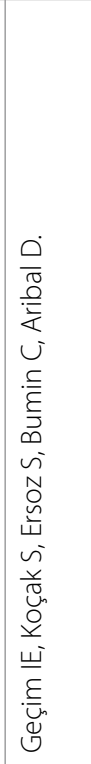 & 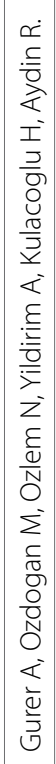 & 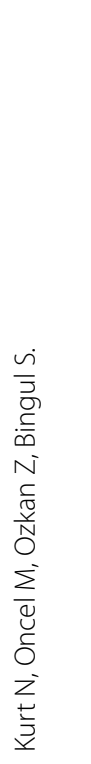 & 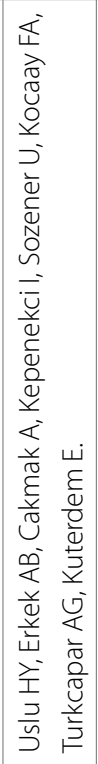 & 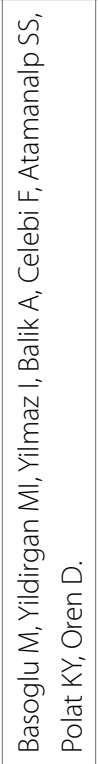 & 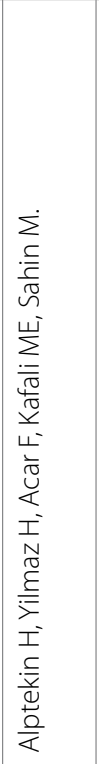 & 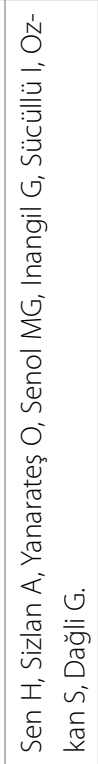 & 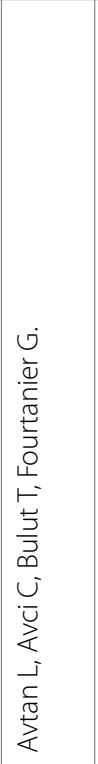 & 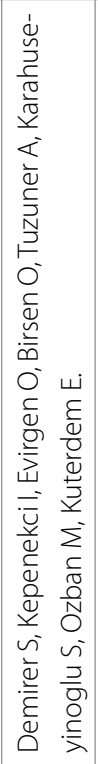 & 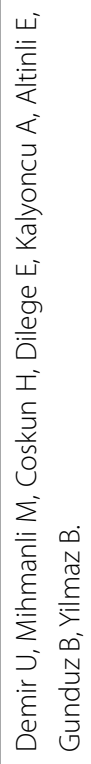 \\
\hline & - & $\sim$ & $m$ & $\nabla$ & & 0 & $\lambda$ & $\infty$ & $a$ & $\stackrel{\circ}{\circ}$ & $\mp$ & & $\stackrel{m}{m}$ & $\stackrel{\Xi}{ \pm}$ & $\stackrel{n}{\sim}$ & & $\approx$ \\
\hline
\end{tabular}




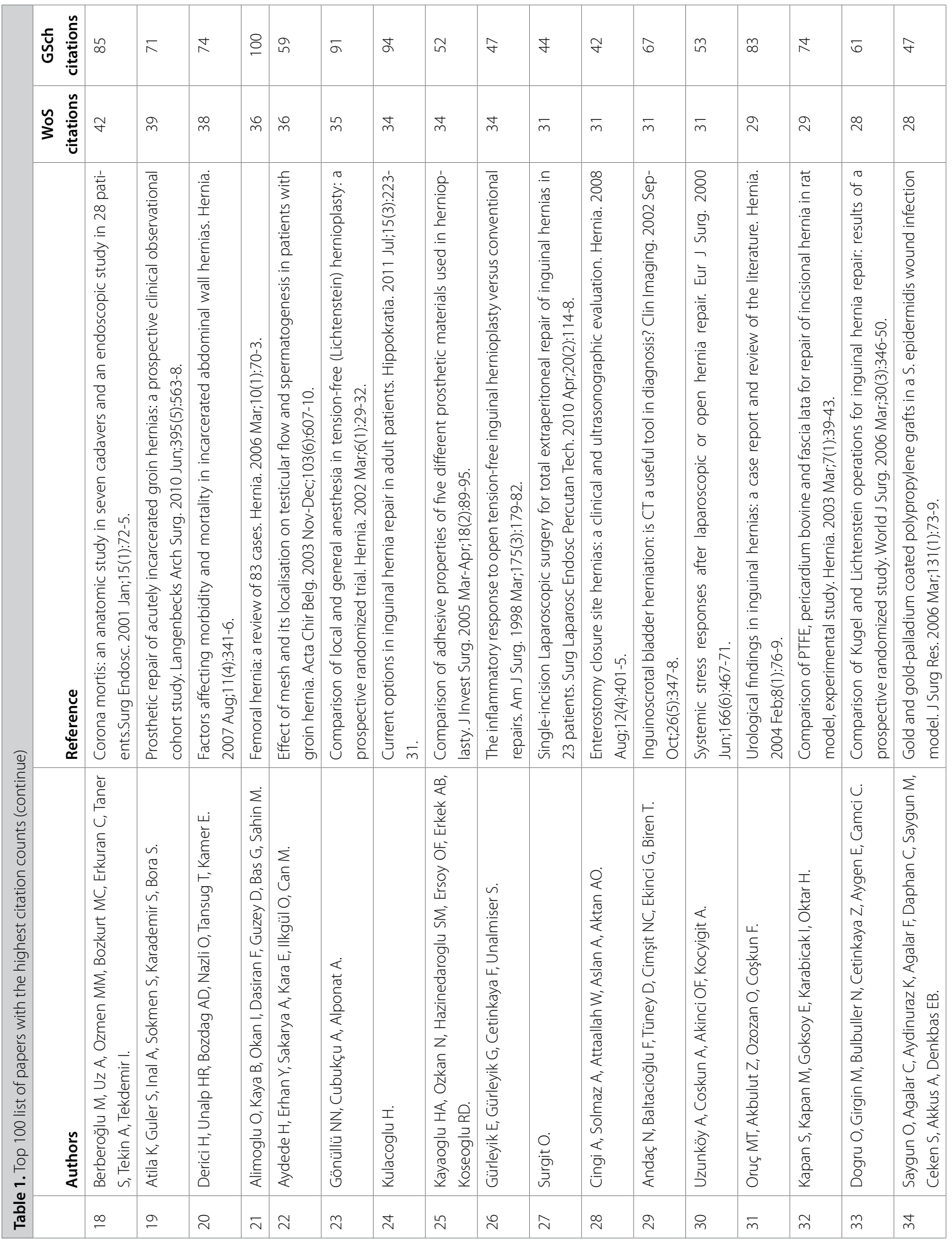




\begin{tabular}{|c|c|c|c|c|c|c|c|c|c|c|c|c|c|c|c|c|}
\hline 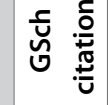 & மீ & $\stackrel{\infty}{\curvearrowleft}$ & f & gr & $\stackrel{\infty}{m}$ & 年 & $\stackrel{m}{m}$ & ìn & $\stackrel{\infty}{m}$ & $\stackrel{\infty}{+}$ & $\hat{m}$ & $\hat{m}$ & $\overline{6}$ & $\stackrel{\infty}{m}$ & $\bar{\sigma}$ & $\stackrel{g}{q}$ \\
\hline 足 & $\stackrel{\infty}{\sim}$ & $\stackrel{\infty}{\sim}$ & $\lesssim$ & $\approx$ & $\lesssim$ & 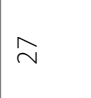 & 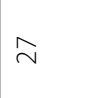 & 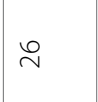 & $\stackrel{\bullet}{\sim}$ & $\stackrel{\bullet}{\sim}$ & 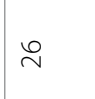 & $\stackrel{\mathscr{N}}{\sim}$ & $\stackrel{\Delta}{\sim}$ & $\stackrel{\Delta}{\sim}$ & $\stackrel{\Delta}{\sim}$ & $\stackrel{ \pm}{\sim}$ \\
\hline 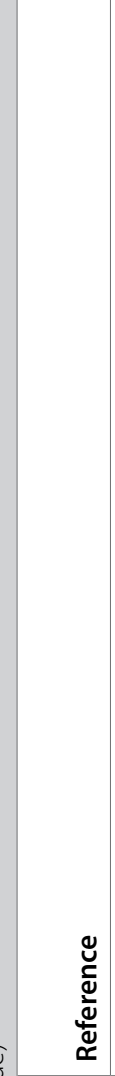 & 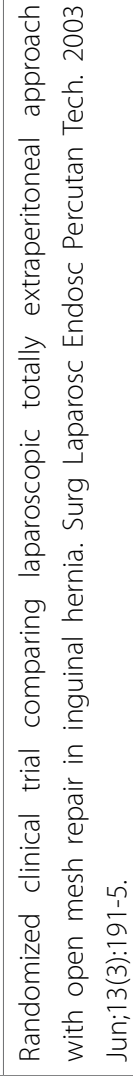 & 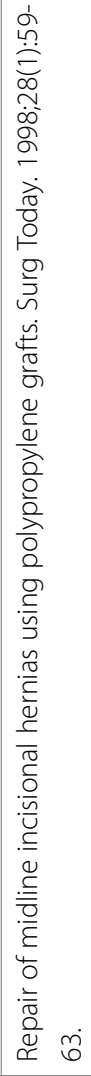 & 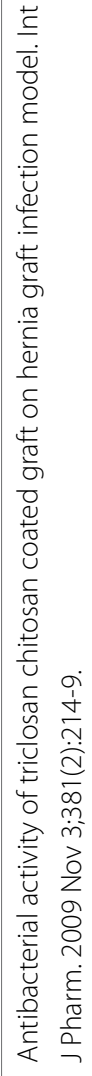 & 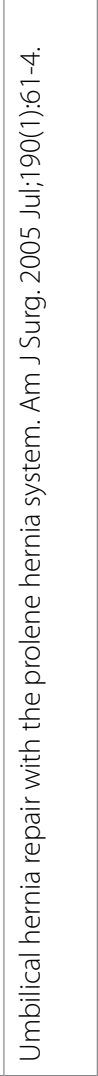 & 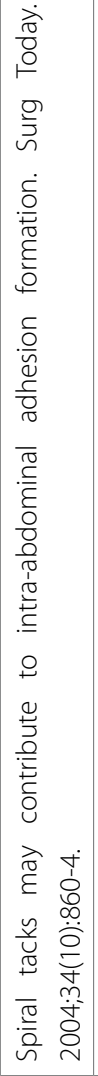 & 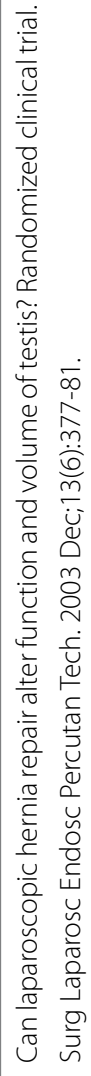 & 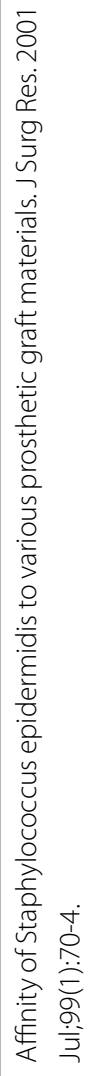 & 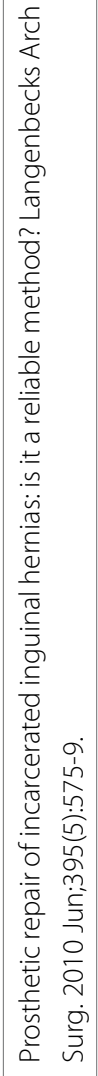 & 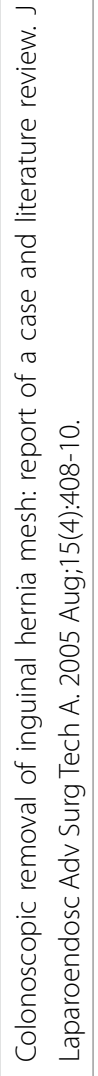 & 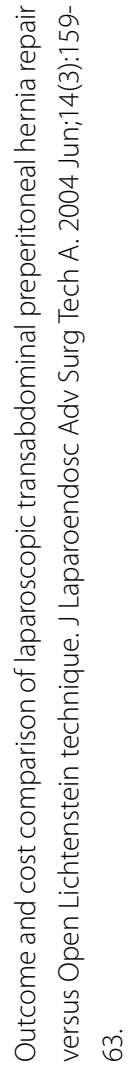 & 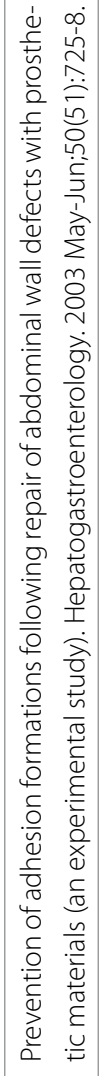 & 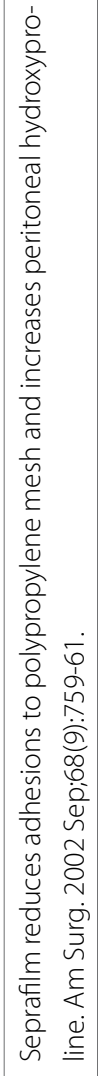 & 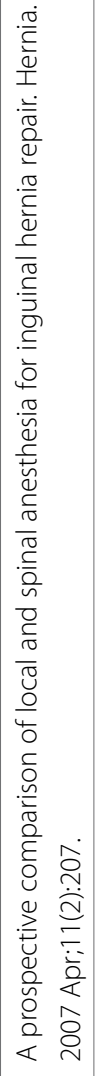 & 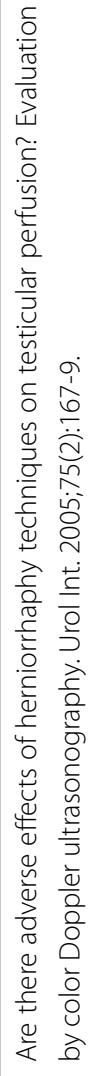 & 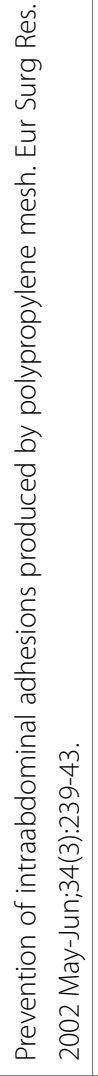 & 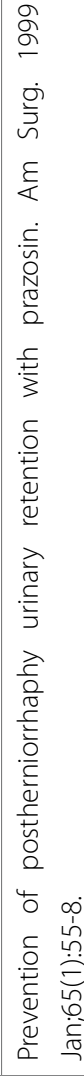 \\
\hline 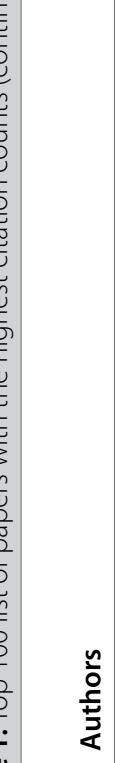 & 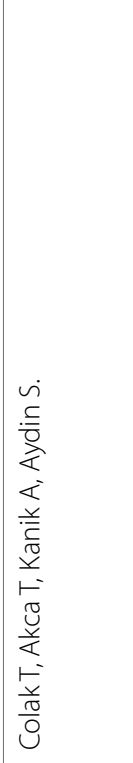 & 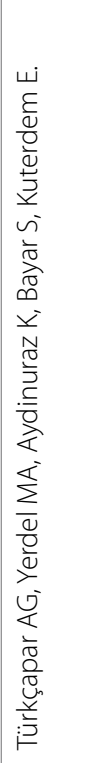 & 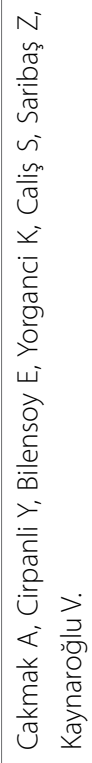 & 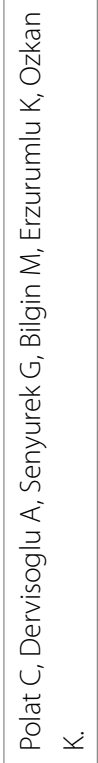 & 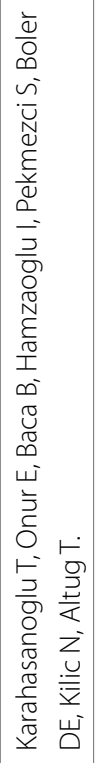 & 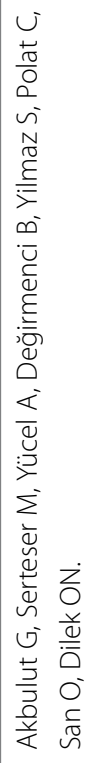 & 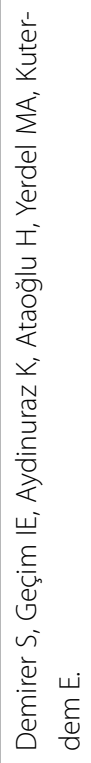 & 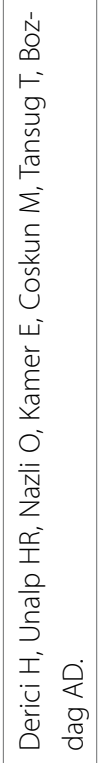 & 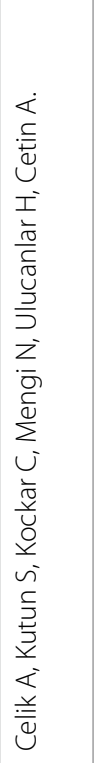 & 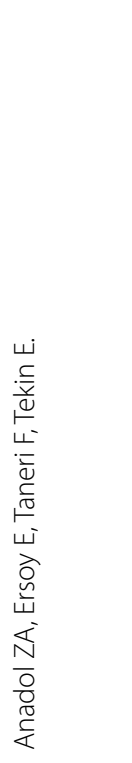 & 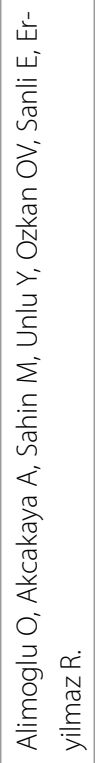 & 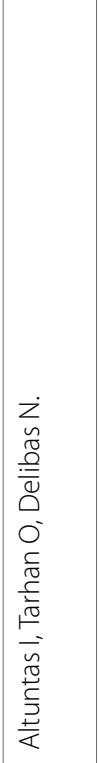 & 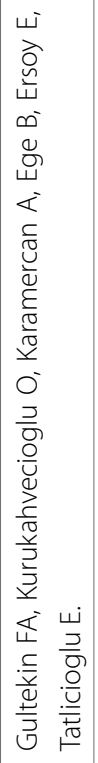 & 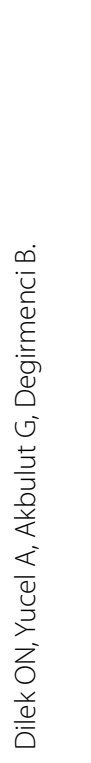 & 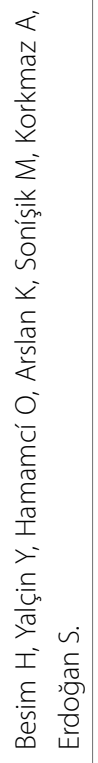 & 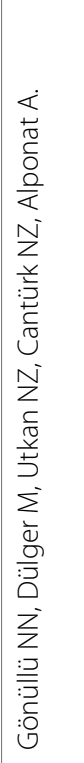 \\
\hline & $\stackrel{\stackrel{n}{m}}{m}$ & $\stackrel{m}{m}$ & $\hat{m}$ & $\stackrel{\infty}{m}$ & $\stackrel{m}{m}$ & q & $\bar{\gamma}$ & F & $\stackrel{m}{q}$ & 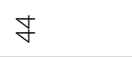 & 占 & o & f & $\stackrel{\infty}{q}$ & $\stackrel{g}{q}$ & ค \\
\hline
\end{tabular}




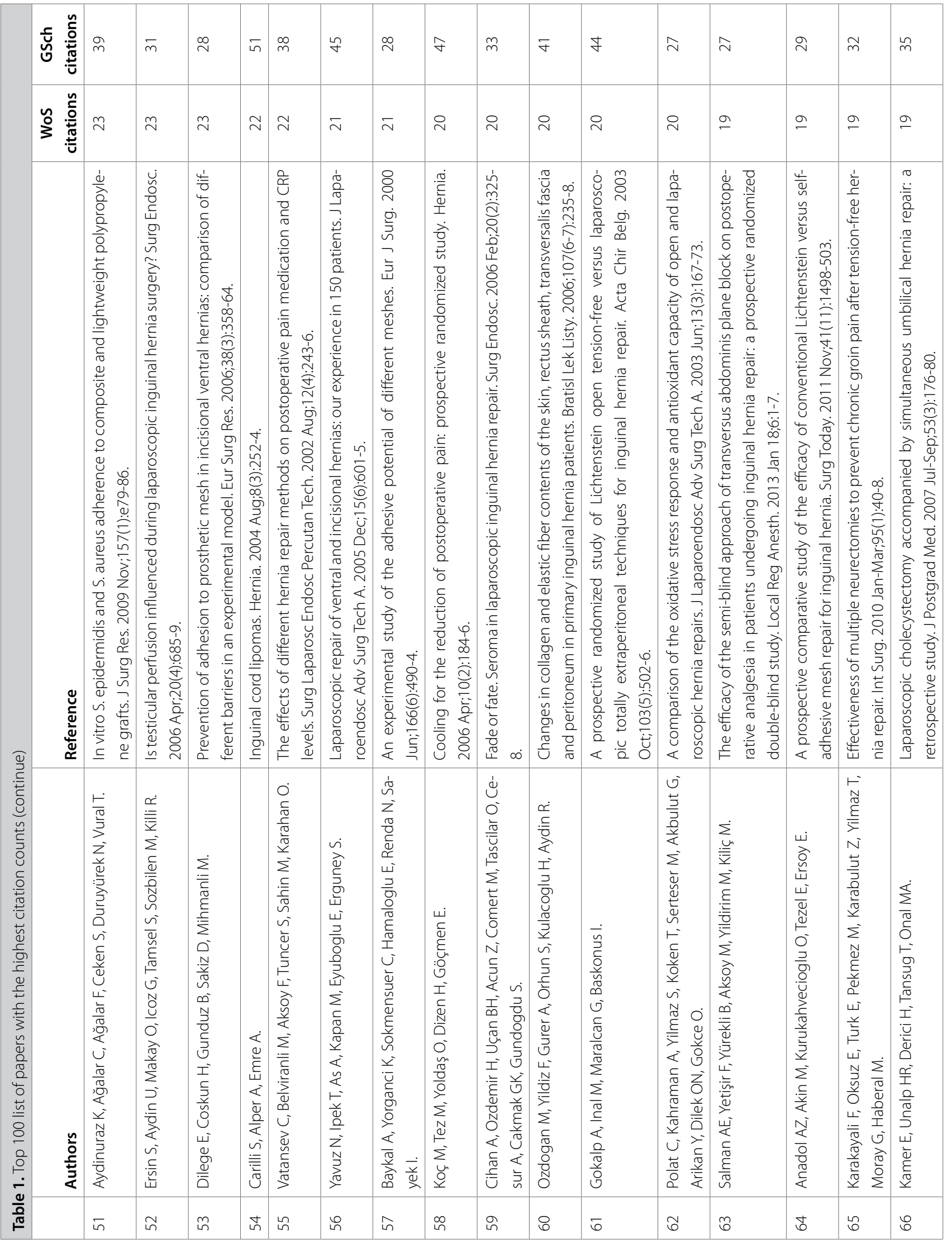




\begin{tabular}{|c|c|c|c|c|c|c|c|c|c|c|c|c|c|c|c|c|}
\hline 点 & 아 & \& & $\hat{m}$ & $\stackrel{\infty}{m}$ & 年 & $\hat{m}$ & ন & ஓి & $\bar{m}$ & $\stackrel{\searrow}{\sim}$ & ஓ & $\stackrel{m}{m}$ & $\stackrel{m}{m}$ & $\stackrel{\searrow}{\sim}$ & $\tilde{m}$ & q \\
\hline 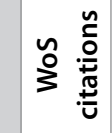 & $\stackrel{\sigma}{\sigma}$ & $\stackrel{\rho}{\sigma}$ & $\stackrel{\Omega}{-}$ & $\stackrel{q}{\sigma}$ & $\stackrel{\infty}{\sim}$ & $\stackrel{\infty}{\sim}$ & $\stackrel{\infty}{\sim}$ & $\stackrel{\infty}{\sim}$ & $\approx$ & $\approx$ & $\stackrel{ }{ }$ & 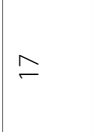 & $\approx$ & $\approx$ & $\stackrel{\bullet}{\sim}$ & $\stackrel{\bullet}{\simeq}$ \\
\hline 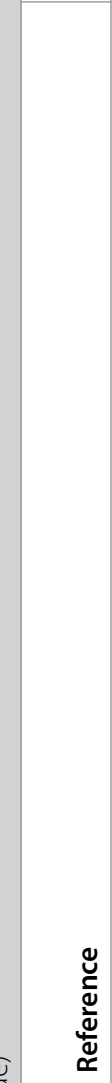 & 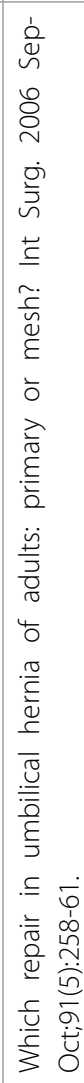 & 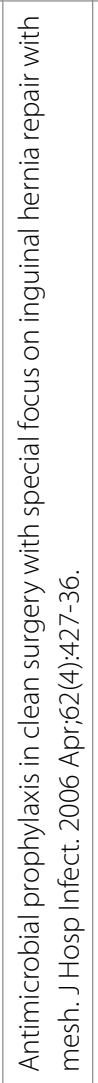 & 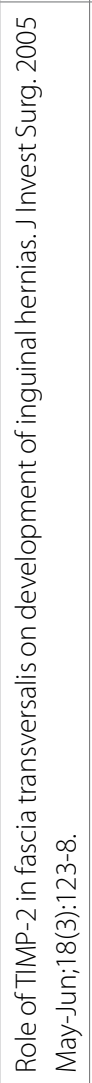 & 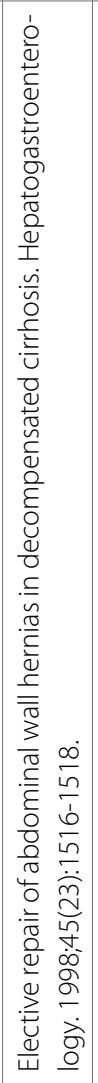 & 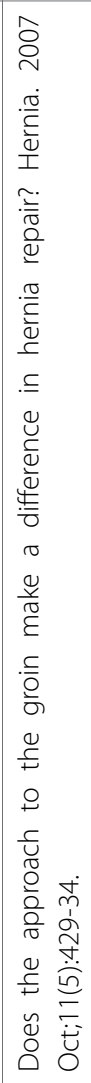 & 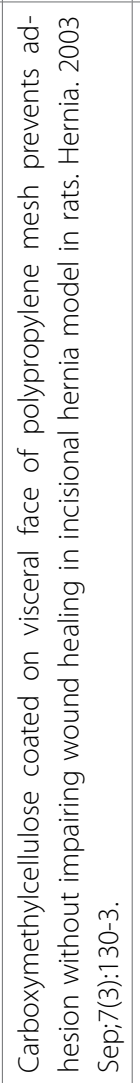 & 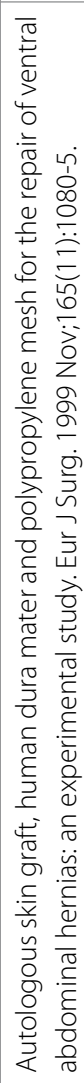 & 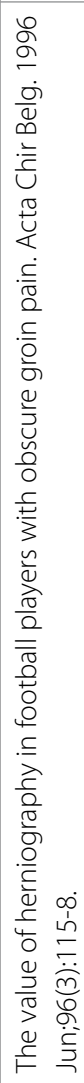 & 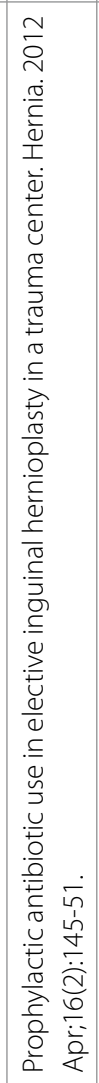 & 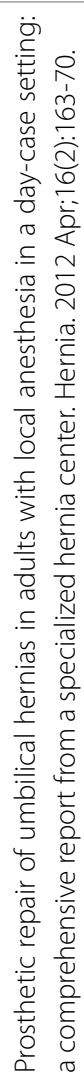 & 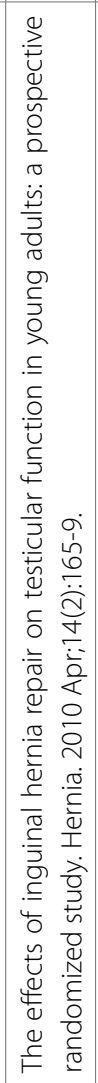 & 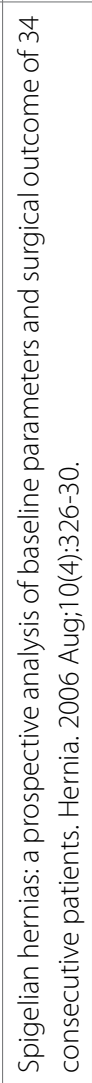 & 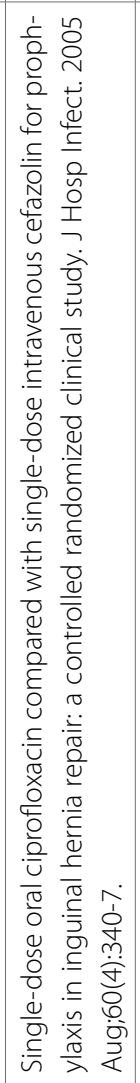 & 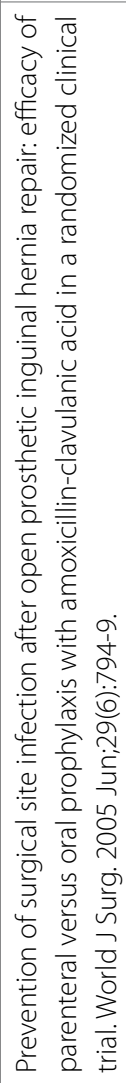 & 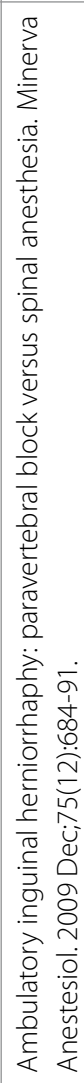 & 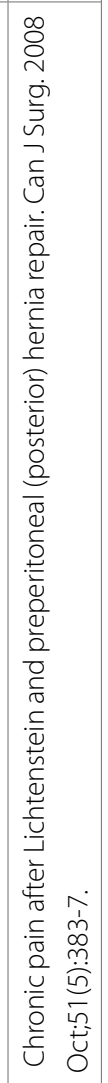 \\
\hline 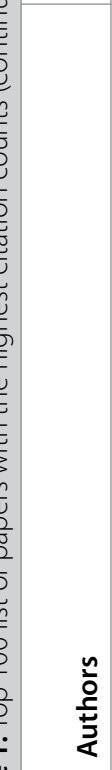 & 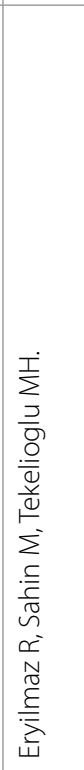 & $\underset{\bar{N}}{\stackrel{0}{0}}$ & 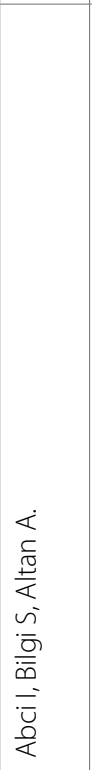 & 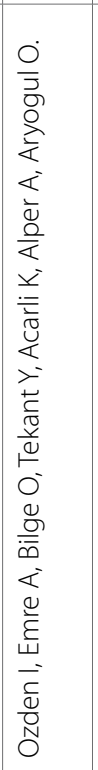 & 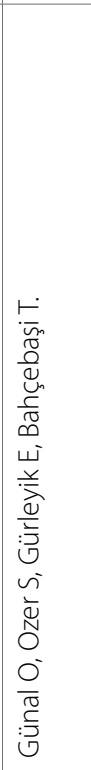 & 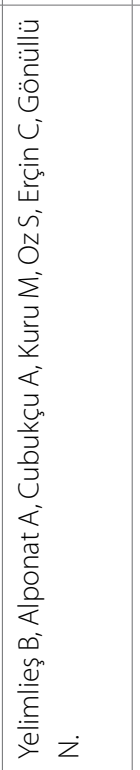 & 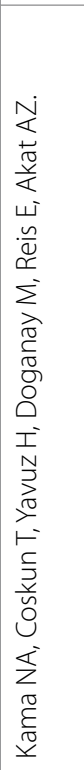 & 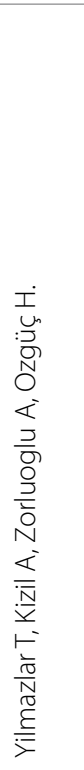 & 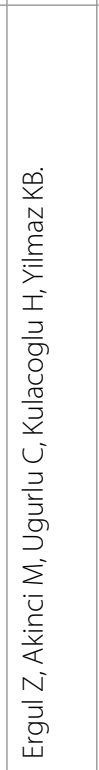 & 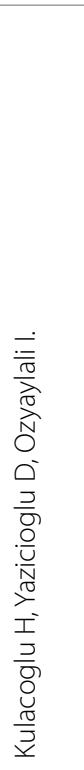 & 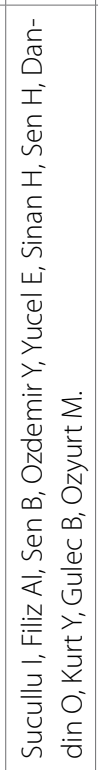 & 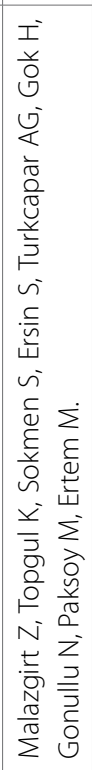 & 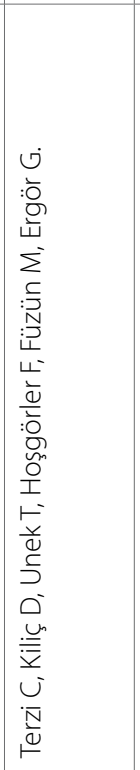 & 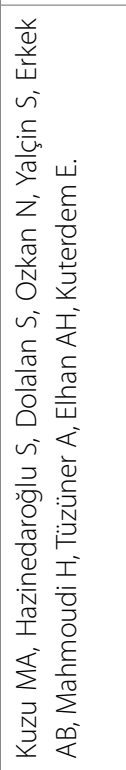 & 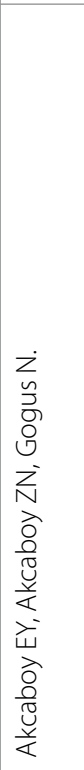 & 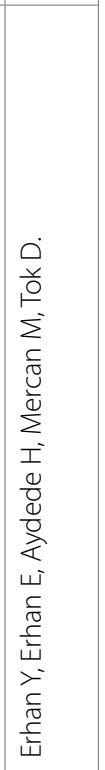 \\
\hline & $\hat{\sigma}$ & $\varnothing^{\infty}$ & 요 & $尺$ & $\Sigma$ & $\approx$ & 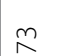 & ㅊ & $\stackrel{n}{\wedge}$ & $\stackrel{\circ}{\Upsilon}$ & $\AA$ & $\stackrel{\infty}{\wedge}$ & g & & $\bar{\infty}$ & $\infty$ \\
\hline
\end{tabular}




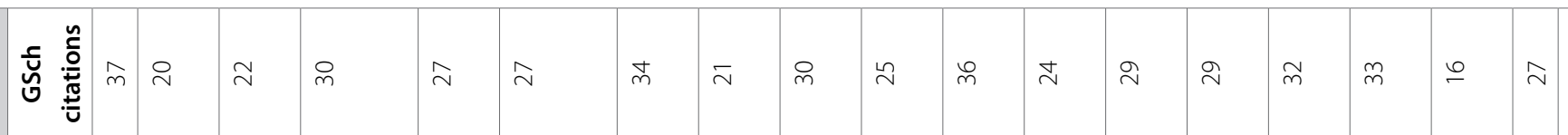

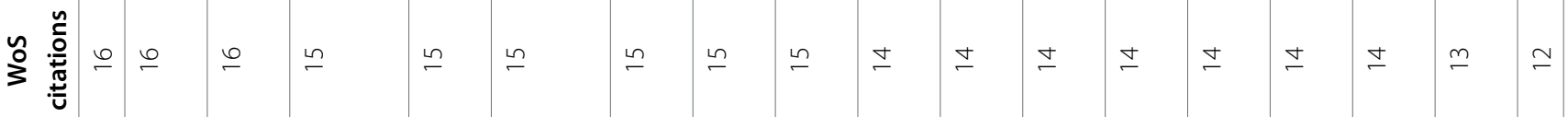

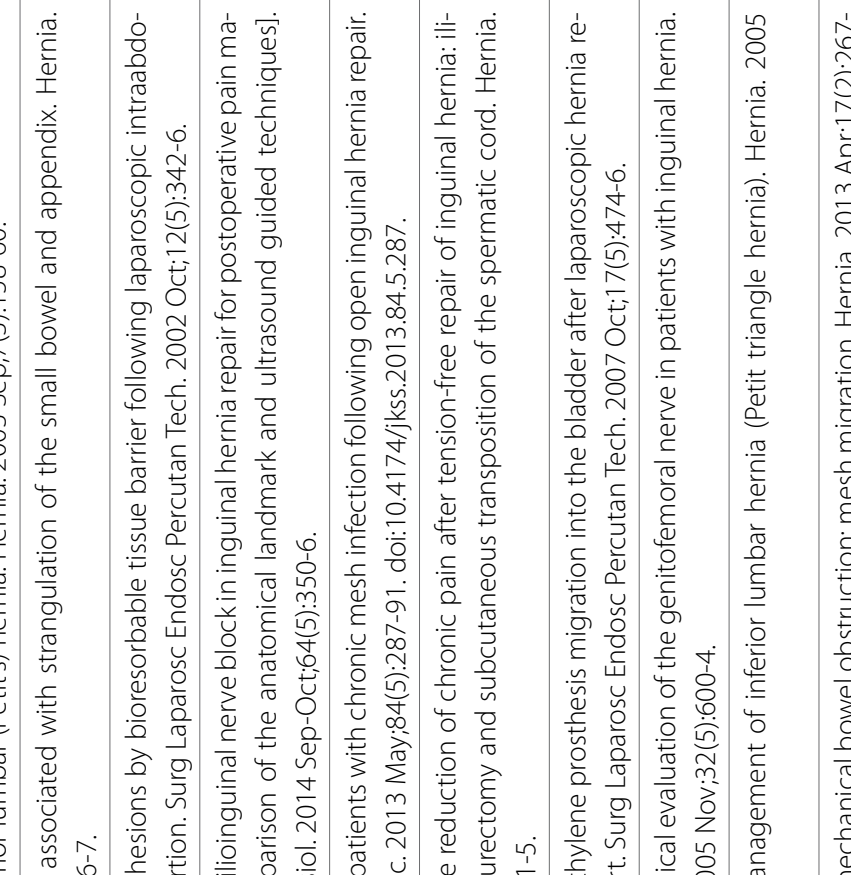

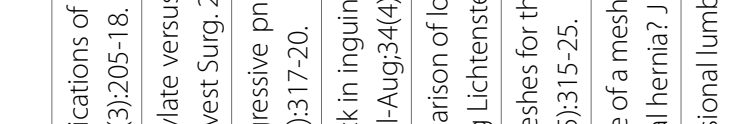
离

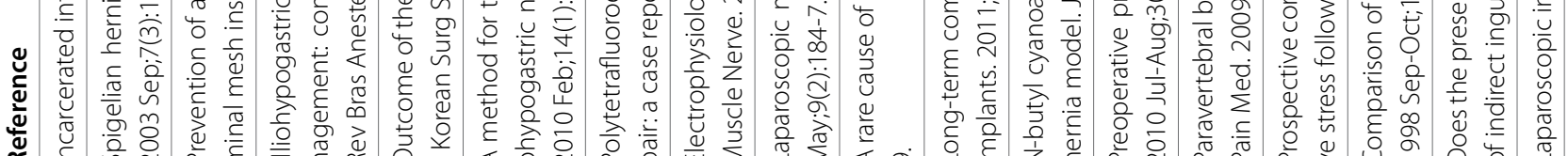

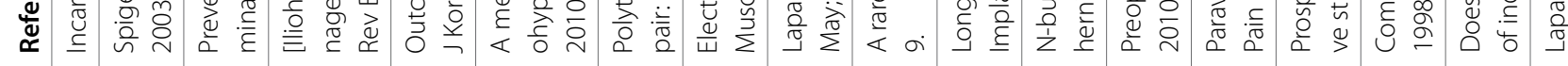

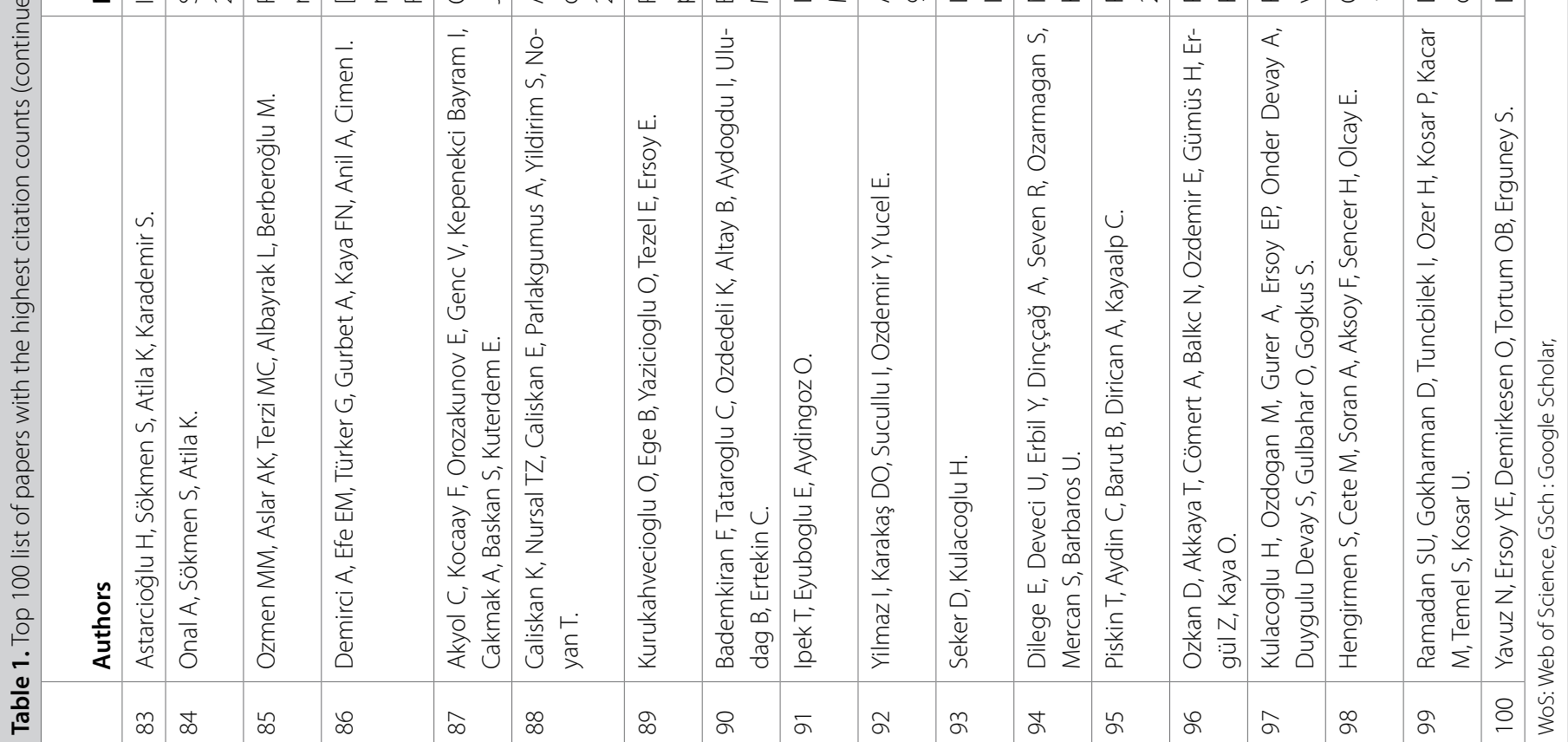


Table 2. Number of articles regarding journal type and decade of publication

\begin{tabular}{|l|c|c|c|}
\hline Decade & Surgery & Others & Total \\
\hline $1990-1999$ & 8 & 1 & 9 \\
\hline $2000-2009$ & 58 & 14 & 72 \\
\hline $2010-2019$ & 14 & 5 & 19 \\
\hline Total & 80 & 20 & 100 \\
\hline
\end{tabular}

Table 3. Mean citations counts of top 100 papers regarding decade of publication

\begin{tabular}{|l|c|c|c|}
\hline Decade & No of papers & WoS citations & GSch citations \\
\hline $1990-1999$ & 9 & $30.00(270)$ & $53.1(478)$ \\
\hline $2000-2009$ & 72 & $32.26(2.323)$ & $60.69(4.370)$ \\
\hline $2010-2019$ & 19 & $24.05(457)$ & $43.37(824)$ \\
\hline p & & 0.35 & 0.27 \\
\hline \multicolumn{2}{|l}{ The figures in parenthesis are total numbers within the decade. }
\end{tabular}

Table 4. Leading journals in the "Top 100 List" in herniology

\begin{tabular}{|l|l|}
\hline Journal name & No of papers \\
\hline Hernia & 23 \\
\hline Journal of Laparoendoscopic Advanced Surgical Techiques and Videoscopy & 6 \\
\hline Surgical Laparoscopy Endoscopy \& Percutaneous Techniques & 6 \\
\hline Journal of Surgical Research & 5 \\
\hline Surgery Today & 5 \\
\hline Acta Chirurgica Belgica & 4 \\
\hline American Journal of Surgery & 4 \\
\hline European Journal of Surgery & 4 \\
\hline Journal of Investigative Surgery & 4 \\
\hline World Journal of Surgery & 3 \\
\hline Surgical Endoscopy & 3 \\
\hline & \\
\hline
\end{tabular}

Table 5. Citation counts regarding type of article

\begin{tabular}{|l|c|c|c|}
\hline Study type & No of papers & WoS citations & GSch citations \\
\hline Clinical study & 66 & 33.05 & 62.27 \\
\hline Review & 4 & 33.00 & 72.00 \\
\hline Case report or case series & 10 & 20.90 & 39.70 \\
\hline Laboratory studies, animal experiments, cadaver study & 20 & 26.40 & 43.85 \\
\hline p & & 0.310 & 0.156 \\
\hline
\end{tabular}


Table 6. Topics of the article

\begin{tabular}{|l|l|}
\hline Inguinal hernia & 58 \\
\hline Incisional hernia & 16 \\
\hline Umbilical hernia & 5 \\
\hline Femoral hernia & 1 \\
\hline Spigelian hernia & 2 \\
\hline Lumbar hernia & 2 \\
\hline Emergency repairs & 6 \\
\hline Mesh & 21 \\
\hline Fixing material & 2 \\
\hline Infection & 11 \\
\hline Some articles focused on multiple topics. & \\
\hline
\end{tabular}

Table 7. Most influential authors with more than 200 citations in total in the "Top 100 List" in herniology

\begin{tabular}{|l|c|c|c|c|}
\hline Institution & $\begin{array}{c}\text { Total number of } \\
\text { citations }\end{array}$ & $\begin{array}{c}\text { Total number of } \\
\text { articles }\end{array}$ & $\begin{array}{c}\text { Highest citation count } \\
\text { for an article }\end{array}$ \\
\hline Ankara University School of Medicine & 401 & 8 & 50.13 & 145 \\
\hline Ankara Numune Teaching Hospital & 366 & 10 & 36.60 & 104 \\
\hline İstanbul University School of Medicine & 366 & 10 & 36.60 & 91 \\
\hline Ankara Atatürk Teaching Hospital & 117 & 4 & 29.25 & 64 \\
\hline 9 Eylül Üniversity & 107 & 5 & 21.40 & 39 \\
\hline Gazi University School of Medicine & 84 & 4 & 21.00 & 26 \\
\hline İzmir Atatürk Teaching Hospital & 83 & 3 & 27.67 & 38 \\
\hline Dışkapı Yıldırım Beyazıt Teaching Hospital & 79 & 3 & 25.67 & 34 \\
\hline Kocaeli University School of Medicine & 77 & & & 35 \\
\hline
\end{tabular}

top 100 list can also enter the list in, and there may be changes in the ranking of some papers already on the list. Also, the citation and publication numbers of the authors in top 100 lists may not thoroughly reflect their overall productivity in those fields.

van Noorden et al. have reported that $43.8 \%$ of all scientific publications have collected no citations at all, and $1.84 \%$ of all stay in the citation band of 100-999 citations (13). Amongst articles in herniology from Turkey, only two papers have passed the 100 citations threshold, and other 13 papers have collected more than 50 citations. Mean number of citations in WoS is only 30. In 2002, Paladugu et al. have reported that the mean citation number of the 100 citation classics in general surgery journals is 405 (4). There was no article on abdominal wall hernias in that top 100 list. A very recent bibliometric study in general surgery has found a median number of 490 citations within the 5 journals with the highest impact factor (14). On the other hand, Mayir et al. have reported that $7.7 \%$ of the most cited articles in general surgery from Turkey was on inguinal hernias (5). Onat has detected 6 papers from general surgery field in a list of 271 articles for Turkey's contribution to medicine, and only one of those was related to the abdominal wall hernias (2). That paper was produced by the Surgical Department of Ankara University School of Medicine (15). Its citation count had been 76 that time and was found to reach 145 in the present search.

The top 100 articles in the herniology field were produced by 43 institutions. Five of the 10 most productive institutions were complied with Nayir et al.'s list for general surgery publications from Turkey (5). Moreover, the most productive city was Ankara in both studies, and non-academic teaching hospitals exhibited as great success as university hospitals did. This difference probably originated from the nature of the hernia surgery, which is suitable for almost all surgical facilities. On the contrary, vast majority of the publications on more complex surgical procedures like transplantation is produced by university hospitals (16). Onat's study on citation counts of Turkish papers from all disciplines revealed a different picture, $90 \%$ of the medical publications in that list were produced by university hospitals (2). A 
bibliometric study for orthopedic publications originating from Turkey concordantly revealed that only one out of the most productive 10 institutions was non-academic teaching hospital (17); it was Ankara Numune Training and Research Hospital, which is also in the second rank in the present study.

The 100 top-cited papers were published in 38 journals. This figure ranges between 10 and 46 in previous publications on citation analyses in different fields $(7-9,18,19)$. Top 100 papers in general surgery was published in only 10 surgical journals (4). In the present study, 80 articles were published in surgical journals, and these articles collected more citations than those in the journals from other disciplines. As a specific journal in its field, Hernia journal receives a large number of submissions related to abdominal wall hernias. It is clearly the top journal in the present study. Unfortunately, no single article published in a journal from Turkey entered the top 100 list.

Journal impact factor is a reflection of the average number of citations to recent articles published in journals. A positive correlation between the journal impact factor and the citation counts is an expected finding. Accordingly, some studies have shown that journal impact factors are strong predictors for citation counts $(7,11,12,19,20)$. However, no correlation was detected between the journal impact factors and the number of the citations in the present study.

Clinical studies are the most frequent article types in the previous bibliometric analyses for citation counts $(10,11,18)$. Unlike, in a bibliometric study on hepatocellular carcinoma articles revealed that review articles collected higher mean number of citations than that for other types (18). In the present study, clinical studies and review articles received more citations than case reports and laboratory studies; however, the difference was not significant. Retrospective clinical series collected more citation counts than prospective studies, possibly because of their larger number of patients from the archives of tertiary reference hospitals than prospective randomized studies with small number of subjects.

Classification of the articles in the top 100 list regarding the topic of the study revealed interesting results. The number of papers on inguinal hernias were more than three times of those about incisional hernias. This is somewhat expected given the fact that inguinal hernia repairs comprise the majority of operations for abdominal wall hernias $(21,22)$. However, incisional hernia articles had a higher mean number of citations than papers on inguinal hernias. On the other side, the total number in the list and the mean citation counts for papers about umbilical hernias are lower than those for incisional hernias despite the fact that the share of these two types of hernias within surgical repairs are quite similar. One reason for this situation may be the complexity in repair of incisional hernias together with more frequent and more serious complications following them.

\section{CONCLUSION}

Citation counts of hernia related articles from Turkey are relatively low. Hernia is the leading journal for Turkish studies. Inguinal hernia is the most frequent topic, whereas papers about incisional hernias receive more citations than others.

Ethics Committee Approval: Not relevant.

Peer-review: Externally peer-reviewed.

Author Contributions: Consept - H.K.; Design - H.K; Supervision - H.K.; Data Collection and/or Processing - H.K., H.C.; Analysis and Interpretation - H.K.; Literature Search - H.C.; Writing Manuscript - H.K.; Critical Reviews - H.K., H.C.

Conflict of Interest: The authors have no conflicts of interest to declare.

Financial Disclosure: The authors declared that this study has received no financial support.

\section{REFERENCES}

1. Yurtsever $E$, Gulgoz S. The increase in the rate of publications originating from Turkey. Sciontometrics 1999; 46: 321-36. [CrossRef]

2. Onat A. Turkey's contribution to medicine: main institutions, fields and publications. Ulus Cerrahi Derg 2013; 29: 105-14. [CrossRef]

3. Garfield E. 100 citation classics from the Journal of the American Medical Association. JAMA 1987; 257: 52-9. [CrossRef]

4. Paladugu R, Schein M, Gardezi S, Wise L. One hundred citation classics in general surgical journals. World J Surg 2002; 26: 1099-105. [CrossRef]

5. Mayir B, Bilecik T, Doğan U, Koç Ü, Ensari CÖ, Oruç MT. Most cited articles in general surgery from Turkey. Ulus Cerrahi Derg 2015; 31: 85-9. [CrossRef]

6. Kulacoglu H, Oztuna D. Growth and trends in publications about abdominal wall hernias and the impact of a specific journal on herniology: a bibliometric analysis. Hernia 2011; 15: 615-28. [CrossRef]

7. Shuaib W, Costa JL. Anatomy of success: 100 most cited articles in diabetes research. Ther Adv Endocrinol Metab 2015; 6: 163-73. [CrossRef]

8. Montinaro V, Giliberti M, Villani C, Montinaro A. Citation classics: ranking of the top 100 most cited articles in nephrology. Clin Kidney J 2019; 12: 6-18. [CrossRef]

9. Varzgalis M, Bowden DJ, MC Donald CK, Kerin MJ. A bibliometric analysis of the citation classics of acute appendicitis. Ann Med Surg (Lond) 2017; 19:45-50. [CrossRef]

10. Ahmad SS, Ahmad SS, Kohl S, Ahmad S, Ahmed AR. The hundred most cited articles in bariatric surgery. Obes Surg 2015; 25: 900-9. [CrossRef]

11. Shuaib W, Khan MS, Shahid H, Valdes EA, Alweis R. Bibliometric analysis of the top 100 cited cardiovascular articles. Am J Cardiol 2015; 115: 972-81. [CrossRef]

12. Brinjikji W, Klunder A, Kallmes DF. The 100 most-cited articles in the imaging literature. Radiology 2013; 269: 272-6. [CrossRef]

13. van Noorden R, Maher B, Nuzzo R. The top 100 papers: Nature explores the most-cited research of all time. Nature 2014;514: 550-3. [CrossRef]

14. Manuel Vazquez A, Latorre Fragua R, Lopez Marcano A, Ramiro Perez C, Arteaga Peralta V, de la Plaza-Llamas R, Ramia JM. The top 100: a review of the most cited articles in Surgery. Cir Esp 2019; 97: 150-5. [CrossRef] 
15. Yerdel MA, Akin EB, Dolalan S, Turkcapar AG, Pehlivan M, Gecim IE, et al. Effect of single-dose prophylactic ampicillin and sulbactam on wound infection after tension-free inguinal hernia repair with polypropylene mesh: the randomized, double-blind, prospective trial. Ann Surg 2001; 233: 26-33. [CrossRef]

16. Bas K, Dayangac M, Yaprak O, Yuzer Y, Tokat Y. International collaboration of Turkey in liver transplantation research: a bibliometric analysis. Transplant Proc 2011; 43: 3796-801. [CrossRef]

17. Gürbüz Y, Süğün TS, Özaksar K. A bibliometric analysis of orthopedic publications originating from Turkey. Acta Orthop Traumatol Turc 2015; 49: 57-66. [CrossRef]

18. Hwang JW, Kim H, Lee DJ. The 100 most influential manuscripts on hepatocellular carcinoma: a bibliometric analysis. J Int Med Res 2019; 47: 1467-82. [CrossRef]
19. Siddiq K, Akbar HF, Khan M, Siddiqui AA, Nusrat S, Blay JY. The 100 most influential papers and recent trends in the field of gastrointestinal stromal tumours: a bibliometric analysis. Cureus 2018; 10: e2311. [CrossRef]

20. Uthman OA, Okwundu Cl, Wiysonge CS, Young T, Clarke A. Citation classics in systematic reviews and meta-analyses: who wrote the top 100 most cited articles? PLoS One 2013; 8: e78517. [CrossRef]

21. Seker G, Kulacoglu H, Öztuna D, Topgül K, Akyol C, Çakmak A, et al. Changes in the frequencies of abdominal wall hernias and the preferences for their repair: a multicenter national study from Turkey. Int Surg 2014; 99: 534-42. [CrossRef]

22. Dabbas N, Adams K, Pearson K, Royle G. Frequency of abdominal wall hernias: is classical teaching out of date? JRSM Short Rep 2011; 2: 5. [CrossRef]

\title{
ORIJINAL ÇALIŞMA-ÖZET
}

Turk J Surg 2020; 36 (2): 180-191

\section{Türkiye'den yayınlanan karın duvarı fıtıkları ile ilgili bilimsel makaleler içinde en çok atıf alan 100 makale: bibliyometrik çalışma}

\author{
Hakan Kulaçoğlư ${ }^{1}$, Haydar Celasin ${ }^{2}$ \\ ${ }^{1}$ Ankara Fitık Merkezi, Cerrahi Bölümü, Ankara, Türkiye \\ ${ }^{2}$ Lokman Hekim Akay Hastanesi, Cerrahi Bölümü, Ankara, Türkiye
}

\section{ÖZET}

Giriş ve Amaç: Bu çalışmanın amacı, Türkiye'den karın duvarı fıtıkları ile ilgili yayınlanan makaleler içinde en çok atıf alan 100 yazıyı bulmak ve değerlendirmektir.

Gereç ve Yöntem: Mart 2019 tarihinde Web of Science veri tabanında yapılan tarama ile en çok atıf alan 100 makale belirlendi. Bu makaleler yayınlandıkları dergilere, yayın tarihine, yazarlarına, makale türüne, makalenin odaklandığı konuya ve yayını yapan merkeze göre analiz edildi. Tüm makalelerin Google Scholar'daki atıf sayıları da kaydedildi.

Bulgular: Listedeki 100 makalenin ortalama atıf sayısı 30,50 idi. Makaleler 38 ayrı dergide yayımlanmıştı, en çok makale Hernia dergisinde yer almıştır. Dergilerin etki faktörü ile atıf sayısı arasında ilişki saptanmamıştır. Yazıların 2/3'ü klinik çalışmalardır. Makale türünün atıf sayısı üzerine etkisi yoktu. Kasık fıtıkları 58 makale ile en sık incelenen konuydu. Kesi fıtıkları ile ilgili yayınlar diğer konulara göre daha fazla ortalama atıf sayısına sahipti. En yüksek atıf alan makalenin yayınlandığı Ankara Üniversitesi Tıp Fakültesi aynı zamanda listedeki yayınlar dahilinde en yüksek toplam atıf sayısına ve makale başına en yüksek atıf sayısına sahip merkezdi. Ankara Numune Eğitim ve Araştırma Hastanesi ve İstanbul Üniversitesi Tıp Fakültesi listeye en yüksek sayıda makale veren kurumlardı.

Sonuç: Fıtıklarla ilgili Türk makaleleri nispeten düşük atıf sayılarına sahipti. En çok makalenin yayınladığı dergi Hernia idi. Kasık fıtıkları çalışmalarda en çok incelenen konuydu, ancak kesi fıtıkları ile ilgili yazıların ortalama atıf sayısı daha yüksekti.

Anahtar Kelimeler: Fıtık, karın duvarı, bibliometrik, atıf

Doi: $10.5578 /$ turkjsurg. 4536 